\title{
Die Linguistik ist anders geworden
}

Wolfgang Klein

Gibt es eigentlich eine Partei der Leute, die sich nicht sicher sind, recht zu haben? Da würde ich gern Mitglied werden.

Camus

\section{Einleitung}

Man gesteht es sich ja ungern ein, aber der Höhepunkt der sprachwissenschaftlichen Forschung in Deutschland lag zweifellos im neunzehnten Jahrhundert. Die Werke der Bopp, Grimm, Humboldt, Paul, Brugmann sind Klassiker, sie werden nach wie vor von den Gelehrten des In- und Auslandes zitiert, mehr wahrscheinlich als jedes linguistische Werk deutscher Zunge, das im zwanzigsten Jahrhundert erschienen ist. Das ist ein Kompliment für unsere Urgroßväter, aber keines für unsere Großväter, für unsere Väter, für uns. Immerhin, eines kann man schon festhalten: seit Mitte der Sechzigerjahre hat die sprachwissenschaftliche Forschung hierzulande wiederum einen erheblichen Aufschwung genommen, in der damaligen DDR etwas früher als in der (alten) Bundesrepublik. Ursprünge und weiterer Verlauf dieser Entwicklung sind verwickelt, und je nachdem, welcher Richtung man anhängt, kann man sie auch ganz unterschiedlich sehen. Der Gang der Wissenschaft folgt ja selten den Vorstellungen der Wissenschaftstheorie, er hat zahllose Momente des Zufalls, ebenso viele Ungerechtigkeiten, und das Ergebnis gleicht einer chaotisch wachsenden Großstadt, in der ständig etwas Neues gebaut, vieles halbgebaut stehen gelassen und selten etwas abgerissen wird und in der die vielen darin Tätigen unablässig nach schwer durchschaubaren Prinzipien hin und her wuseln. Sie alle verfolgen ihre Pläne; aber man hat nicht den Eindruck, dass es gelungen ist, „die verschiednen Mitarbeiter in der Art, wie die gemeinschaftliche Absicht erfolgt werden soll, einhellig $\mathrm{zu}$ machen: so kann man immer überzeugt sein, daß ein solches Studium bei weitem noch nicht den sicheren Gang einer Wissenschaft eingeschlagen, sondern ein bloßes Herumtappen sei." (so Immanuel Kant am Beginn der „Kritik der reinen Vernunft”).

Aber aus einem gewissen Abstand betrachtet, zeichnen sich doch auch einige auffällige Züge in dieser Entwicklung seit Mitte der Sechzigerjahre ab. Drei dieser Züge 
möchte ich im Folgenden betrachten. Dies sind der Hang zum Englischen, die Ablösung von der philologischen Tradition und die Rolle von formalen Methoden. Alle drei Momente der Entwicklung kann man ganz unterschiedlich bewerten; ich selbst sehe sie mit gemischten Gefühlen, und ich will deutlich zu machen suchen, warum.

\section{Wir sind ,,international"}

\subsection{Weg von der Muttersprache}

Anfang der Sechzigerjahre war die sprachwissenschaftliche Forschung in Deutschland im Wesentlichen traditionelle, mehr oder minder solide Philologie mit einem klaren Schwergewicht auf der Erforschung der älteren Sprachstufen. Dies gilt für die Germanistik wie für die anderen Philologien unter Einschluss der Indogermanistik, mit der die Allgemeine Sprachwissenschaft in der Regel institutionell verbunden war. Wenn es denn überhaupt eine ausgeprägte theoretische Linie gab, dann die der ,inhaltbezogenen Sprachwissenschaft", vertreten durch Wissenschaftler wie Leo Weisgerber, Jost Trier, Helmut Gipper und andere; selbst frühe Strukturalisten wie Hans Glinz sind zumindest zeitweilig unter den Einfluss dieser Richtung geraten. Der Kerngedanke dieser Betrachtungsweise war, dass die Sprache, in der man aufgewachsen ist und in der man zu denken und zu handeln gelernt hat, die „Muttersprache" also, den „Zugriff auf die Welt" prägt. Der Gedanke ist nicht neu, er wird im Allgemeinen Wilhelm von Humboldt zugeschrieben und hat in Bejamin Lee Whorff seinen radikalsten Vertreter gefunden (es ist, ganz am Rande, bemerkenswert, dass Whorff außerhalb der Sprachwissenschaft selbst einer der bekanntesten und meistzitierten Sprachwissenschaftler ist, ein ausgesprochener Fall von Buitengeltung). Mir selbst ist weder damals noch heute recht klar geworden, was darunter eigentlich zu verstehen ist. Als meine Muttersprache empfinde ich den rheinfränkischen Dialekt, den ich bis zum zehnten Lebensjahr gesprochen habe, und ich habe nicht den Eindruck, dass er meine Vorstellungen über das Wirkliche in entscheidender Weise prägt. Auf der anderen Seite kann man auch nicht in Abrede stellen, dass man sich mit der Sprache auch ein gewisses System von Begriffen, Unterscheidungen und Normen aneignet, dem sich zu entziehen zumindest nicht leicht ist und die daher möglicherweise auch die Art und Weise bestimmen, in denen man nicht zuletzt Wissenschaft betreibt.

Wie immer dies sein mag: nicht nur ist die ,inhaltbezogene Sprachwissenschaft" weithin von der Bildfläche verschwunden - das Deutsche selbst ist dabei, als Wissenschaftssprache zu verschwinden. In den Naturwissenschaften ist dieser Zustand bereits 
erreicht. In anderen Fächern wird sich unser heimisches Idiom sicher etwas länger halten, etwa in der Jurisprudenz, in der die Primärtexte einstweilen noch auf Deutsch vorliegen, oder in der Wissenschaft von der deutschen Sprache selbst, der Germanistik. Aber auch die Germanistik entwickelt sich außerhalb der deutschsprachigen Länder selbst mit rasanter Geschwindigkeit zu einem Nischenfach, und jene, die es in den USA, in Frankreich oder in Skandinavien noch studieren, schreiben immer weniger auf Deutsch. Für die Linguistik gilt es ebenso, wie man durch einen Blick auf die Veröffentlichungen leicht feststellen kann. Man mag diese Entwicklung beklagen oder pathetisch beschwören. Vor allem aber ist es ein massiver Nachteil - ein „Selektionsnachteil", um es in der Sprache der Biologie zu formulieren - für jene, die hierzulande Wissenschaft betreiben. Wer auf Deutsch schreibt, dessen Arbeiten haben allenfalls Binnengeltung und oft nicht einmal diese. Von meinen eigenen Veröffentlichungen werden die englischen stärker rezipiert als die deutschen; das ist vielleicht nicht verwunderlich; verwunderlich ist aber, dass dies selbst in Deutschland so ist.

\subsection{Ein Selektionsnachteil}

Oft wird die mörderische Politik der Nazizeit und die Auswirkungen des letzten Krieges für den Niedergang der deutschen Forschung und, damit verbunden, des Deutschen als Wissenschaftssprache verantwortlich gemacht. Das ist naheliegend; aber es ist nicht sonderlich plausibel. Zum einen setzt, wie die schönen Untersuchungen von Ulrich Ammon gezeigt haben, die rückläufige Entwicklung schon in den Zwanzigerjahren ein. Das gilt für die Sprachwissenschaft nicht anders als für andere Fächer, mit Ausnahme vielleicht der Physik und einiger weniger geisteswissenschaftlicher Fächer. Wen gibt es denn zu Beginn der Dreißigerjahre noch, den man mit Bopp, Grimm oder Paul vergleichen könnte? Vielleicht Karl Bühler - aber er hat es, wiewohl seit 1938 in den Vereinigten Staaten, nie über Binnengeltung hinaus gebracht. Zum anderen haben Vertreibung, Verfolgung und Krieg in der Tat zu einem Kahlschlag an Universitäten und sonstigen Forschungsstätten geführt. Aber das gilt für die Wirtschaft nicht minder als für die Wissenschaft. Während sich die Wirtschaft aber in wenigen Jahren erholt hat, gibt es nicht nur kein „Wissenschaftswunder", sondern einen stetig fortschreitenden Niedergang, was Rang und internationale Geltung der deutschen Forschung angeht - vielleicht nicht in allen, aber doch in fast allen Fächern. Zum Dritten schließlich ist dieser Niedergang für die meisten Disziplinen - nicht jedoch die Linguistik - erst in den letzten zwanzig Jahren zum freien Fall geworden, also dreieinhalb Jahrzehnte nach dem Ende des Krieges. Man kann daher für das 
allmähliche Verschwinden des Deutschen als Wissenschaftssprache nicht die Zeit bis 1945 verantwortlich machen. Die Ursachen müssen anderer Art sein.

Ich denke, es gibt im Wesentlichen zwei Gründe. Der eine ist, dass die Forschung hierzulande in den meisten Disziplinen nicht mehr so gut ist wie früher, und der zweite ist, dass ein Wissenschaftler, der auf Deutsch schreibt, halt nicht mehr von denen gelesen wird, von denen er gelesen werden möchte. Die erste dieser beiden Behauptungen mag etwas schroff klingen, und sie bedarf sicher der Differenzierung. Für die Linguistik beispielsweise trifft sie nicht zu. Nun gut, ich bin ein Linguist und möchte mein Fach hochhalten; aber es ist leicht zu sehen, dass die Linguistik hierzulande international wesentlich stärker rezipiert wird als vor zwanzig, dreißig oder vierzig Jahren - freilich nicht so wie die deutsche Sprachwissenschaft im 19. Jahrhundert. Aber im Übrigen kann man einfach nicht übersehen, dass die Ergebnisse unserer Forschungen im Durchschnitt wenig internationalen Widerhall haben, dass deutschsprachige Lehrbücher, wo nicht ohnehin aus anderen Sprachen übersetzt, immer weniger von hierzulande erzielten Ergebnissen, von hierzulande entwickelten Theorien berichten, dass es immer schwieriger wird, in Deutschland guten Nachwuchs zu finden. Nehmen wir an, man würde durch eine weltweite Umfrage unter den Fachwissenschaftlern erkunden, wer die zehn derzeit führenden Forscher ihrer Disziplin sind - Physiker, Biologen, Chemiker, Informatiker, Psychologen, Archäologen, Anthropologen, Kunsthistoriker, was immer (die Linguistik lasse ich einmal außen vor). Ich glaube, dass sich auf nur wenigen dieser Listen ein Deutscher fände. Das hat vielleicht mit dem mangelnden Geschick in der „Vermarktung" zu tun - aber es wäre töricht zu glauben, es hinge nicht auch an der Qualität des Produkts. Es gibt allen Grund zu der Befürchtung, dass sich in einigen Jahren sogar unter den zwanzig besten kaum noch Deutsche finden. Ist dies für die Linguistik anders? Einen gewissen Anlass zu gedämpftem Optimismus gibt es vielleicht.

Mag man sich über die erste der beiden obigen Behauptungen noch streiten - über die zweite, über das Verschwinden des Deutschen als Wissenschaftssprache, kann es keinen vernünftigen Zweifel geben. Vor sechs Jahren hatte ich einmal Gelegenheit, mit zwei älteren Mitgliedern der Schwedischen Akademie der Wissenschaften, jener Einrichtung, die die Nobelpreise vergibt, zu Abend zu essen. Zu meiner großen Freude konnten beide die „Lorelei" tadellos aufsagen; sie haben aber beide bekundet, keine deutsche wissenschaftliche Literatur auf ihrem jeweiligen Fachgebiet mehr zu lesen. Der Unterschied zu den jetzt aktiven Wissenschaftlern liegt darin, dass diese, in Schweden wie anderswo, auch die „Lorelei" nicht mehr aufsagen können. Sie haben gar kein Deutsch mehr gelernt oder vielleicht ein paar Brocken, aber sicher nicht 
genug, um sich die Mühe zu machen, deutsche wissenschaftliche Arbeiten zu lesen. Dafür gibt es zwei ganz unabhängige Ursachen. Die eine hat nichts mit dem Deutschen im Besonderen zu tun, es ist das allgemeine Vordringen des Englischen in vielen Lebensbereichen - eine Entwicklung, die freilich in Deutschland besonders groteske Züge annimmt. Man versteht nicht recht, weshalb manche Fernsehsender ihre Nachrichten nunmehr „news" nennen und die Telekom ihre Stadtgespräche „city call”, obwohl die Mehrzahl ihrer Kunden so gut wie kein Englisch kann. Nur die Computerexperten nennen die Computer noch Rechner. Die zweite Ursache liegt darin, dass es in so gut wie allen Ländern der Welt immer weniger Interesse an deutscher Sprache und Kultur gibt. Abgesehen von den Verbrechen der Nazizeit gibt es eigentlich kaum deutsche Themen, die ein weltweites Echo finden, und dies ist kein Thema, das den ohnehin mühseligen Erwerb der deutschen Sprache besonders attraktiv machen würde. Warum - um Himmels willen - sollte jemand freiwillig Deutsch lernen? Es ist schon bemerkenswert, wie sehr wir an kultureller Attraktivität gegen das Wilhelmische Zeitalter zurückgefallen sind.

Die meisten Naturwissenschaftler, die ich kenne, regen sich über die hier beschriebene Entwicklung nicht sonderlich auf. Sie schreiben halt auf Englisch, und warum auch nicht? Schließlich hat es manche Vorzüge, wenn sich alle Wissenschaftler derselben Sprache bedienen. Lange war dies das Lateinische, jetzt, nach ein paar Jahrhunderten ohne klare Festlegung und mit regem Gebrauch einiger Nationalsprachen, ist es das Englische. Der Vergleich täuscht jedoch. Das Latein war, zu diesen Zeiten jedenfalls, für alle eine Zweitsprache; das Englische ist für manche eine Zweitsprache, für andere die Muttersprache. Diese Asymmetrie hat drei massive Folgen.

Die erste ist ein gewisser Bruch mit der geistigen Tradition. Ein solcher Bruch kann unterschiedliche Auswirkungen haben. In manchen Fällen ist er vielleicht heilsam. In vielen Disziplinen, beispielsweise in der Astrophysik oder in der Biochemie, macht er sich nur wenig geltend. In anderen aber ist er, jenseits aller kulturkritischen Beschwörungen, einfach ein Desaster, weil die wissenschaftliche Arbeit oft nicht so sehr darin besteht, neue Fakten zu ermitteln, sondern die Fakten in bestimmter Weise $\mathrm{zu}$ interpretieren und in einen bestimmten geistigen Zusammenhang zu stellen. Solche Deutungen sind eng an kulturelle und sprachliche Traditionen gebunden, deren Preisgabe das Ende einer sinnvollen und gut legitimierten Form der Wissenschaft bedeutet.

Die zweite negative Folge ergibt sich aus der mangelnden Beherrschung der Zweitsprache im Vergleich zur eigenen. Der alte Jux „The language of science is bad English" täuscht darüber hinweg, dass dies für manche Disziplinen einen massiven Selektionsnachteil bildet. Das gilt sicher nicht für die Mathematik oder die Chemie, 
wo man zur Not vielleicht auch den verbindenden Text zwischen den Formeln weglassen könnte. Es gilt aber sehr wohl für all jene Disziplinen, in denen der Begrifflichkeit, überhaupt der sprachlichen Form, in der die Erkenntnisse und Theorien ausgedrückt werden, ein höheres Gewicht zukommt - sagen wir der Soziologie, der Kunstgeschichte, der Anthropologie, aber auch der Psychologie oder der Entwicklungsbiologie. Und nur ein Narr könnte sich darüber hinwegtäuschen, dass dieser Nachteil sich bei der Verbreitung der eigenen Ansichten massiv geltend machen kann - weniger vielleicht noch bei Veröffentlichungen, in denen man sich den Text redigieren lassen kann, als bei Vorträgen und Diskussionen. Wie viele Vorträge habe ich gehört, bei denen der Vortragende gute Sachen zu sagen hatte, aber nicht im geringsten landen konnte, weil sein Englisch hinter dem der muttersprachlichen Sprecher so weit zurückgefallen ist! Nicht die Wissenschaft, aber der Wissenschaftsbetrieb ist auch ein Markt, in dem es leider nicht nur auf die Qualität des Produkts ankommt, sondern auch auf die Art seiner Präsentation.

Die dritte negative Folge ist mit Abstand die wichtigste: es entsteht eine Kluft zwischen der Sprache wissenschaftlicher Arbeiten und der Sprache der akademischen und kulturellen Umwelt, in der diese Arbeiten entstehen. Am klarsten zeigt sich dies in der Lehre. Linguistische Seminare, an denen der akademische Unterricht weiterhin auf Deutsch ist, sind für ausländische Studenten natürlich nur dann zugänglich, wenn sie Deutsch lernen - ganz egal, in welcher Sprache die dort Lehrenden publizieren. Dafür haben aber in der Regel nur Auslandsgermanisten einen besonderen Anlass und offenbar auch die immer weniger. Wenn das Englische die Sprache der Wissenschaft ist, dann werden die besten Studenten bei sonst gleichen Bedingungen natürlich bevorzugt dorthin gehen wollen, wo das ganze Umfeld englischsprachig ist. Mit dem Verlust des Deutschen als Wissenschaftssprache können wir die besten Leute immer schwerer halten, soweit sie hier geboren sind, noch gar anziehen, soweit sie aus anderen Ländern kommen. Dies gilt für Studenen wie für Dozenten. Es heißt nicht unbedingt, dass keine ausländischen Studenten oder Dozenten mehr nach Deutschland kommen wollen; es werden aber in der Regel nicht die besten sein. All dies lässt erwarten, dass Rang und Ansehen der deutschen Wissenschaft in der Welt rasch weiter absinken werden. Dies gilt für die Universitäten allgemein, es gilt vielleicht auch für die linguistischen Fächer im Besonderen.

Nun ist dieser Zug abgefahren. Wir sind ,international", wir müssen es sein. Aber man sollte sich nicht darüber hinwegtäuschen, dass dies seinen Preis hat. 


\section{Es gibt viele Wissenschaften von der Sprache}

Dem Gedanken der Philologie, der „Liebe zum Wort", liegt die Idee zugrunde, dass es ja doch viele Gemeinsamkeiten, vielleicht sogar eine Einheit auf all jenen Gebieten gibt, die sich mit den verschiedenen Sprachen und ihren Hervorbringungen, den Texten, beschäftigen. Vor dreißig, vierzig Jahren aber haben sich an den Universitäten die meisten Philologien in einzelne Teilbereiche, etwa in Literaturwissenschaft, Linguistik und Mediävistik und manchmal noch andere, aufgeteilt. Wenn man sich den Gang der Wissenschaften seit der Antike betrachtet, erscheint ein solches Auseinandertreten ganz natürlich. Zu Beginn war alles Philosophie, und die fortschreitende Erweiterung der Kenntnisse über die Jahrtausende hat $\mathrm{zu}$ der reichen Struktur der Wissenschaften geführt, die wir heute kennen. Die Wissenschaft von der Sprache und den Werken der Sprache ist, gemessen an den Wissenschaften von der Natur und ihren Werken, ein Spätentwickler, und sie hat nach wie vor nicht die Reife jener erreicht. Man sieht es allein schon daran, dass ein jeder, der da lesen und schreiben kann, glaubt, etwas über Sprache und Literatur sagen zu können, und dies vielleicht nicht einmal ohne Grund.

Es ist nun eigentümlich, dass die einzelnen Naturwissenschaften, Biologie, Chemie, Physik, wie immer man sie einteilt, in gewisser Weise doch wiederum eine sind; biologische Prozesse sind chemische, und chemische Prozesse sind physikalische, und die Gesetze dieser sind die Gesetze jener, wenn auch der Grad der Auflösung, mit der man die Erscheinungen beschreibt, verschieden ist, und so eine jede Disziplin ihr Recht hat. Warum eigentlich sollte dies für jene Fächer anders sein, die sich mit der Struktur und Funktion der Sprache, mit ihrer historischen Entwicklung oder mit den Eigenschaften sprachlicher Werke, vom Hildebrandslied bis zur „Publikumsbeschimpfung", vom Weihnachtslied bis zum Werbetext befassen und ihnen ihre Geheimnisse $\mathrm{zu}$ entreißen versuchen? Eigentümlicherweise ist zwischen jenen, die sich mit diesen verschiedenen Seiten der Sprachlichkeit beschäftigen, aber nicht bloß das Bewusstsein verloren gegangen, dass letztlich alles von denselben Prinzipien bestimmt ist, so unterschiedlich sich diese im Einzelnen zeigen mögen; ihr Verhältnis zueinander ist auch weithin von Verständnislosigkeit, Gleichgültigkeit, Misstrauen, ja Abneigung gekennzeichnet. Die große Familie, zu der sie doch alle gehören, gleicht den Atriden. Kein Biologe würde den Physiker gering schätzen, noch gilt dies umgekehrt, obwohl die Biologie sicher eine viel „weichere" Disziplin ist, gemessen an den Standards der „harten" Physik. Anders sieht es aus, wenn es um das Verhältnis von Literaturwissenschaftlern und Linguisten geht. Für diesen Unterschied gibt es sicher verschiedene Gründe. Vielleicht haben die Geisteswissenschaftler eher einen 
hämischen Charakter. Aber selbst wenn es so wäre, würde man sich fragen, wie dies kommt. Der wichtigste Grund scheint mir vielmehr zu sein, dass beide Disziplinen, Linguistik wie Literaturwissenschaft, gemessen an den Naturwissenschaften nicht weit genug entwickelt sind. Wir haben unser Wissen ungemein gemehrt. Aber was die Prinzipien angeht, die alles Sprachliche bestimmen, seine Struktur, seine Entwicklung, seine Wirkung in den unterschiedlichen Hervorbringungen, so sind unsere Galileis, Newtons, Lavoisiers noch nicht gekommen. Keiner in unseren Fächern hat diese Prinzipien; aber die Einzelnen unterscheiden sich vielleicht darin, für wie lohnenswert und aussichtsreich sie es halten, danach zu suchen. Gerade deshalb wäre es aber doch sinnvoll, wenn jene, die sich mit der Sprache, ihrer Entstehung, ihrer Entwicklung und ihren verschiedenen Hervorbringungen befassen, an einem Strick zögen.

Sie tun es aber nicht. Sie tun es über die letzten Jahrzehnte immer weniger. Die einschlägigen Einrichtungen in den philologischen Fakultäten sind spezialisiert. Dort, wo es, wie in der Germanistik, der Romanistik, der Slawistik oder den sonstigen Philologien, noch gemeinsame Institute gibt, sind sie in verschiedene Abteilungen getrennt, deren Angehörige sich mit kollegialer Freundlichkeit, manchmal mit Achtung, ebensooft mit Gleichgültigkeit, oft genug mit Abneigung begegnen. Sie lesen einander nicht. Man freut sich über die Ausnahmen, die es gibt, aber man kann sich nicht verhehlen, dass es Ausnahmen sind. Was sind die Gründe dafür?

Da gibt es sicherlich viele. Einer der wichtigsten aber sind zwei gleichsam schon kanonische Vorurteile, die jeweils die eine Seite gegenüber der anderen hat. Nicht wenige Literaturwissenschaftler haben die Vorstellung, dass die Sprachwissenschaft, jedenfalls die moderne, nichts ist als ein unverständlicher, esoterischer Formelkram, der sich von der Realität der Sprache vollkommen gelöst hat. Die Sprache in ihren unterschiedlichsten Erscheinungsformen, vom Gespräch im Wartezimmer bis zur hohen Dichtkunst, ist etwas Fassliches, Konkretes, das die Linguisten in Wäldern von Strukturbäumen verschwinden lassen. Für die Sprache, in der die Linguisten zueinander reden, gilt, was Chamfort über die Sprache der Basken gesagt hat: „Es heißt, dass sie einander verstehen. Ich glaube es aber nicht." Dies ist, vielleicht etwas zugespitzt formuliert, das kanonische Vorurteil der Literaturwissenschaftler gegenüber den Linguisten. Das kanonische Vorurteil der Linguisten gegenüber den Literaturwissenschaftlern ist dasselbe, und wiederum ganz anders: Man kann ihre Auslassungen nicht verstehen, weil es gar nichts zu verstehen gibt. Es bleibt alles im Vagen, nichts lässt sich so recht fassen, es gibt keine Kriterien dafür, wie man das Wahre vom Falschen scheiden kann, es gibt eigentlich keinen sinnvollen Erkenntnisfortschritt, sondern nur Meinungen. 
Schön wäre natürlich zu sagen, dass dies eben bloße Vorurteile sind, die zu überwinden eine wesentliche Aufgabe für uns alle, die sich mit der Sprache befassen, darstellt. Aber so ist es nicht. Ich will diese Meinung, statt lange Argumente beizuziehen, einmal mit der alten Methode der Exempla belegen (die Verfasser sind nicht angegeben, weil es ja beim Exemplum auf das Exemplarische ankommt und nicht auf das Individuelle). Der Literaturwissenschaftler hat Recht mit seinen Vorurteilen:

„We therefore generalize the definition of Move in the obvious way: if Move targets $\mathrm{K}+\{\mathrm{w},\{\mathrm{X}, \mathrm{Y}\}\}$ in the structure $\mathrm{S}$, raising a and merging a with $\mathrm{K}$ to form $\mathrm{L}=\{\mathrm{w},\{\mathrm{a}, \mathrm{K}\}\}$, then $\mathrm{K}$ is replaced by $\mathrm{L}$ wherever it appears in $\mathrm{S}$. If $\mathrm{S}=$ $\mathrm{K}$, we have the special case in which the full structure is targeted and extended by Move. In the example of object raising just given, Move targets $\mathrm{K}=\{\mathrm{AGR}, \mathrm{VP}\}\}$, forming $\mathrm{L}=\{\mathrm{AGR},\{\mathrm{DP}, \mathrm{K}\}\}$, so it automatically modifies $\mathrm{TP}=\{\mathrm{T},\{\mathrm{T}, \mathrm{K}\}\}$ to $\mathrm{TP} @=\{\mathrm{AGR},\{\mathrm{T}, \mathrm{L}\}\}$, modifies $\{\mathrm{AGR}$, $\{$ AGR, TP $\}$ to $\{$ AGR, $\{$ AGR, TP@ $\}$, etc."

Der Linguist hat mit seinen Vorurteilen Recht:

„Damit haben wir interpretierend das innere Gleichgewicht der Hymne erspürt, in dem die strenge Hoheit des Ewigen und der süße Schmelz des seelen- und sinnenhaft Menschlichen wunderbar und geheimnisvoll ausgewogen sind. Der Kreis schließt sich, indem wir erkennen, wie es hier möglich wurde, den ,erfüllten Augenblick" in die mythische Welt selbst hineinzutragen, ohne sie damit aufzuheben, oder, schärfer gefaßt, dem Augenblick den mythischen Erkenntnisumriß einzuprägen, so dass er den zeitentrückten Grund des menschlichen Daseins überhaupt zu spiegeln vermag: das Titanische sowohl wie die offenste Hingabe enthüllen sich darin als nur zwei Seiten desselben Grundverhalts, nämlich der wesenhaften Gebundenheit des Menschen an seine Abstammung vom Alliebenden, oder, wenn wir hier das Gleichnis vom sonnenhaften Auge noch einmal anführen dürfen, seiner Sonnenhaftigkeit."

Natürlich wird jetzt sofort ein Linguist kommen und sagen, dass man diese Formeln wohl verstehen kann. Und ein Literaturwissenschaftler wird kommen und sagen, dass das hier Gesagte alles andere als vage ist und dass es sehr wohl Maßstäbe dafür gibt, 
ob es wahr oder falsch ist. Und der eine glaubt dem anderen nicht, und der andere nicht dem einen.

Da ich nun selbst ein Linguist bin, will ich vielleicht doch das linguistische Vorurteil ein bisschen zurücknehmen (in einem gewissen Sinne wohl auch beibehalten). Mir scheint nämlich, dass es zwar in allen Wissenschaften um das Wahre und das Falsche geht und darum, das Ausmaß und den Anteil des Ersteren zu mehren, dass man aber sehr wohl unterschiedliche Formen des Erkenntnisfortschrittes unterscheiden sollte. Es gibt so etwas wie einen additiven und einen substitutiven Erkennntnisfortschritt in den einzelnen Disziplinen, und zwar in allen Disziplinen, obwohl ihr relativer Anteil schwankt und sich auch im Laufe der Zeit nicht selten verschiebt. Mit substitutivem Erkenntnisfortschritt meine ich, dass, was man auf einem bestimmten Felde zu einer bestimmten Zeit über irgendeinen Gegenstand herausgefunden hat, dasjenige, was man zuvor über diesen Gegenstand geglaubt hat, ersetzt. Das, was zuvor da war, ist damit in gewisser Weise ad acta gelegt, es gehört ins Museum der Wissenschaften, als Kuriosum oder auch als seinerzeit bedeutende, nun aber überwundene Leistung unserer Vorgänger im Geschäft der Erkenntnis. Über Jahrhunderte haben die Menschen geglaubt, dass die Krankheiten in erster Linie durch Dyskrasien der verschiedenen Körpersäfte Zustandekommen, sie haben mit dieser Auffassung bisweilen erstaunliche Heilerfolge erzielt, sie sind oft damit gescheitert, nicht anders als die ärztliche Wissenschaft unserer Tage auch. Niemand aber glaubt heute mehr an die Lehre von den Dyskrasien, sie ist einfach überwunden, durch andere Auffassungen ersetzt, so wie an die Stelle dieser wiederum andere treten werden: die Entwicklung ist substitutiv. Mit einem additiven Erkenntnisfortschritt meine ich, dass neben frühere Erkenntnisse in einem bestimmten Bereich neue, andere treten, die zu jenen entweder überhaupt nicht in einem Gegensatz stehen oder aber dieselbe Sache in gewisser Weise anders betrachten. In beiden Fällen mehren sie gleichfalls unsere Einsicht auf dem jeweiligen Gebiet, ohne jedoch das, was vorher war, gleichsam zu überschreiben und auszulöschen: es besteht gleichberechtigt weiter.

Besonders schön lässt sich der Unterschied am Beispiel des Aristoteles zeigen, der ja sowohl ein Philosoph (im heutigen, engeren Sinne dieses Wortes) wie auch ein bedeutender Naturwissenschaftler war, vielleicht der bedeutendste, der je gelebt hat. Nun würden nur die Wenigsten sagen, dass sich des Aristoteles Ausführungen über die Metaphysik die einschlägigen Ausführungen des Plato als falsch erwiesen haben, so wie er denn selbst durch den Heiligen Thomas widerlegt wurde und dieser durch Descartes und der wiederum durch Kant und Kant schließlich durch Heidegger oder durch Wittgenstein. So sehen wir nicht die Entwicklung der Philosophie seit ihren 
antiken Anfängen. Vielmehr steht uns Plato neben Aristoteles und neben Hume und neben Schelling und neben Husserl, und auch die unter uns lebenden und wirkenden Denker haben sich mit aufs Klassenphoto gestellt. Dennoch kann man sicher nicht sagen, es habe in der langen Geschichte der Philosophie keinerlei Erkenntnisfortschritt gegeben. Er ist nur anderer Art - er war additiv. Nun war „Philosophus" nicht bloß Metaphysiker und Ethiker, sondern auch Naturwissenschaftler. Bertrand Russell meinte sogar, er sei eigentlich überhaupt nur als Biologe bedeutend gewesen, eine Meinung, der ich selbst nicht beitreten kann. Wir neigen allerdings dazu, die Leistungen des Stagiriten auf diesem Gebiet zu vergessen, wahrscheinlich deshalb, weil so vieles, was er über die Entstehung der Fliegen oder über die Zeugung der Tiere oder über die Eigenschaften der Delphine gesagt, einfach falsch ist, obwohl es sich länger vor der ,scientific community", dem selbsternannten obersten Gerichtshof der wissenschaftlichen Wahrheit, behauptet hat als mutmaßlich alles, was den Heutigen als wissenschaftlich erwiesen gilt. Wir sind uns gewiss, dass manche seiner Ansichten unzutreffend waren; sie tauchen nur noch im Kuriositätenkabinett der Wissenschaften auf; sie sind substituiert. Nicht alles freilich, was Aristoteles an biologischen Tatsachen zusammengetragen hat, ist ersetzt; in vielem hat er Recht behalten, und dieses Wissen ist nicht substituiert, sondern bloß ergänzt worden.

Die beiden Formen des Erkenntnisfortschrittes finden sich in allen Wissenschaften; aber ihr Anteil und ihre Bewertung sind höchst verschieden. In der Philosophie ist er im Wesentlichen additiv, in der Biologie, wie in allen Naturwissenschaften, vorwiegend substitutiv. Die Literaturwissenschaft ist in ihrem Kernbereich durch additive Entwicklung des Wissens gekennzeichnet. Es gibt aber freilich sehr wohl Bereiche mit substitutivem Erkenntnisfortschritt; diese Bereiche würde man allerdings eher als Hilfsgebiete ansehen, etwa im Biographischen oder in der Editionsgeschichte. Ob die erste Auflage des „Doktor Faust" von Heine bei Hotop in Kassel oder bei Voigt in Wandsbek erschienen ist, diese Frage ist inzwischen entschieden, eine früher gehegte Auffassung ist widerlegt. Das aber ist ein vergleichsweise randständiges Problem der Disziplin, gemessen etwa an der Frage, wie man den „Doktor Faust" als sprachliches, als literarisches Kunstwerk $\mathrm{zu}$ deuten hat oder wie sein Verhältnis $\mathrm{zu}$ anderen Gestaltungen des Faust-Stoffes zu sehen ist. In diesem Punkt ist der Fortschritt eher von additiver Art: eine Deutung tritt neben eine andere, und alle fühlen sich immer mehr belehrt.

Die Sprachwissenschaft, die ältere wie die neuere, versteht sich hingegen eindeutig als eine Disziplin, in der es im genannten Sinne um substitutiven Fortschritt geht. Die modernen Linguisten, wie schon ihre Vorgänger im neunzehnten Jahrhundert, 
schmeicheln sich, frühere Analysen eines bestimmten Gegenstandsbereiches, sagen wir des indoeuropäischen Konsonantismus oder der Funktion der Tempora, ein für allemal erledigt zu haben: die jetzigen sind richtig, die älteren sind falsch und gehören ins Museum. Dort werden vielleicht auch die heutigen einmal landen, wenn entsprechende Beweise kommen. Dies ist die allgemeine Ansicht, es ist auch die meine. Darin liegt, so scheint mir, vielleicht der massivste Gegensatz zwischen linguistischer und literaturwissenschaftlicher Betrachtungsweise, und er ist ein tieferer Abgrund zwischen den Disziplinen als etwa der unterschiedliche Gebrauch der Sprache, den wir oben am Beispiel der Realität karikiert haben und der vielleicht eher eine Folge dieses eigentlichen Gegensatzes ist.

Als Nicht-Linguist müsste man jetzt sofort fragen: „Wo ist denn nun in der Tat dieser Erkenntnisfortschritt? Was haben die modernen Linguisten in den letzten fünfundzwanzig Jahren beispielsweise über die deutsche Sprache, sei es die der Gegenwart oder der Vergangenheit, Neues herausgefunden, das frühere Analysen widerlegt oder auf eine völlig neue Ebene der Erkenntnis hebt?" Und da gerät man schon etwas ins Schleudern. Als ich diese Frage vor fast zehn Jahren einmal Edwin Williams gestellt habe, hat er nach längerem Nachdenken gemeint: „Die Entdeckung und Klärung des Problems der parasitic gaps'".

Gut, immerhin etwas. Aber was hat man sonst noch herausgefunden, das man in vielleicht etwas aufbereiteter und popularisierter Form, aber doch guten Gewissens der Gemeinschaft der an der Sprache und ihren Gesetzen Interessierten mitteilen könnte? Das ist nicht leicht zu sagen. Es ist zum einen so, dass die Analysen der gegenwärtigen Linguistik außerordentlich subtiler, tiefer, wirklich tiefgründiger sind als vieles, ja das meiste, was man bis vor zwanzig oder dreißig Jahren gemacht hat. Dies gilt für die deutsche Sprache nicht anders als für die anderen Philologien, für die Gegenwartssprache ebenso wie für Probleme der Sprachgeschichte, obwohl letztere vielleicht weniger häufig behandelt werden. Das ist der positive Teil der Antwort. Auf der anderen Seite ist aber vielleicht ja doch relativ wenig an neuem Wissen herausgekommen, das man als ,lexikonfest" bezeichnen könnte. Damit meine ich ein Wissen, das jenen Bestätigungsgrad, jenen Grad an Sicherheit erreicht hat, den man sich von einem guten Lexikon erwartet. Was wirklich wahr ist, können wir nie wissen, und dies widerspräche auch der Idee des substitutiven Erkenntnisfortschrittes: wenn sich das Vergangene als falsch erweisen kann, dann muss sich auch das Gegenwärtige als falsch erweisen können.

Und an solchem lexikonfesten Wissen gibt es nun wenig, mit dem der moderne Linguist aufwarten könnte. Selbstverständlich gibt es über die deutsche oder eine 
andere normalerweise an der Universität studierte Sprache, sei es die der Gegenwart oder einer älteren Sprachstufe, eine Menge an lexikonfestem Wissen. Wir wissen einfach, wie die Kasusmorphologie ist, was die verschiedenen Wortstellungsmuster sind, wir kennen zumindest über weite Strecken die historische Entwicklung. Nur stammen diese Erkenntnisse nicht aus den letzten fünfundzwanzig Jahren, sondern aus früherer Zeit und zu nicht geringen Teilen aus dem neunzehnten Jahrhundert. Der Linguist glaubt an den substitutiven Erkenntnisfortschritt, allein, er ist auf diesem Weg nicht allzusehr fortgeschritten. Woran liegt dies?

Es gibt sicher viele Gründe, von denen mir zwei besonders wichtig zu sein scheinen. Zum einen hat sich nicht nur die Germanistik (wiederum als Beispiel für alle Philologien) in ihrer Gesamtheit in Teildisziplinen wie Mediävistik, Linguistik, (neuere) Literaturwissenschaft aufgegliedert, sondern die Fraktionierung setzt sich in die Linguistik hinein fort. Dies hat die Entwicklung in den letzten Jahren dazu geführt, dass sich die einzelnen Forscher immer mehr als Anhänger einer bestimmten Schule, eines bestimmten theoretischen Rahmens mit spezifischen Annahmen, einer spezifischen Beschreibungssprache und auch spezifischen Formalismen verstehen. Sie untersuchen zwar dieselbe Sprache und deren strukturelle Besonderheiten, aber sie tun dies in ihrer je eigenen Sprache, und die Kommunikation untereinander wird immer schwieriger. Dies führt denn zusehends dazu, dass ein tatsächlicher Erkenntnisfortschritt, wo es ihn denn gibt, innerhalb des jeweiligen ,frameworks" liegt und von anderen gar nicht bemerkt oder nicht verstanden wird. Was die eine Schule für sich entdeckt und vielleicht auch ganz präzise formuliert, das wissen andere vielleicht längst, bloß haben sie es in einem anderen Räderwerk, in einer anderen Beschreibungssprache zu erfassen versucht. Zwischen den einzelnen ,frameworks" sind die Kommunikationskanäle zusehends versandet. In Wirklichkeit ist das Bild, wie man sich vorstellen mag, sehr differenziert; manches lässt sich wohl ineinander übersetzen, manche Richtungen stehen sich nahe, andere fern, manche Vertreter einer bestimmten Schule sind höchst dogmatisch und lehnen es ab, aus ihrem Rahmen Fallendes auch nur zu lesen, andere versuchen sich zumindest ein Bild $\mathrm{zu}$ schaffen, wieder andere wechseln die Schule und dergleichen mehr. Aber die Tendenz ist klar: der Erkenntnisfortschritt ist nicht mehr ein Erkenntnisfortschritt, der allgemein unseren lexikonfesten Wissensstand über beispielsweise die deutsche Sprache beträfe; es ist ein Erkenntnisfortschritt innerhalb einer bestimmten Denkschule; für andere ist es keiner, entweder weil sie längst dort angelangt sind oder weil sie es nicht zur Kenntnis nehmen. Auf einen besonderen Aspekt dieser fachinternen Fraktionierung, nämlich die „Formalisierung" werde ich in Abschnitt 4 ausführlich zurückkommen. 
Der zweite Grund ist, dass sich, vielleicht unvermeidlich beim Fortgang einer Wissenschaft, das Interesse zunehmend von einer breiten Erfassung der sprachlichen Erscheinungen zu einem immer tieferen Bohren an Einzelphänomenen verlagert hat. Man versucht nicht mehr, den gesamten Bereich der deutschen (oder einer anderen) Sprache insgesamt mit den besten Methoden empirisch abzudecken, sondern man wirft sich auf bestimmte Teilbereiche, die immer wieder untersucht werden. Mit einem Schlagwort, die Untersuchung geht von der Breite in die Tiefe. Man findet daher eine Fülle höchst feinsinniger Untersuchungen beispielsweise der Bindung anaphorischer Elemente oder der Extrapositionsmöglichkeiten aus dem Mittelfeld des deklarativen Hauptsatzes. Aber es gibt keine umfassende Grammatik der deutschen Sprache, in der man einfach nachschlagen könnte, was die moderne Linguistik beispielsweise über den deutschen Satz zutage gefördert hat, so dass es nunmehr als gesichertes Wissen gilt - im Vergleich zu dem, was vielleicht vor dreißig, vierzig Jahren bekannt war. Dies gilt keineswegs nur für die Syntax. Ein anderes vielleicht noch schlagenderes Beispiel ist die lexikalische Semantik. In der Semantik insgesamt hat man - zumindest nach meiner Auffassung - in den letzten fünfundzwanzig Jahren ganz erhebliche Fortschritte gemacht. Doch hat sich die Entwicklung auf bestimmte Bereiche, insbesondere auf die Semantik bestimmter syntaktischer Konstruktionen, sagen wir die Bindungseigenschaften anaphorischer Elemente, konzentriert. Was man darüber weithin vernachlässigt hat, ist eine systematische Untersuchung der einzelnen Wörter - Nomina, Verben, Adjektive - in variierenden Kontexten. Es gibt auf der einen Seite konventionelle Wörterbücher, wie man sie mehr oder minder in derselben Form schon vor hundert Jahren hatte; es gibt auf der anderen Seite sehr punktuelle Untersuchungen einzelner lexikalischer oder funktionaler Elemente; was es nicht gibt, ist eine Bedeutungsanalyse umfassender Teile des deutschen Wortschatzes mit Methoden der modernen Semantik. Ich komme darauf noch einmal in vierten Abschnitt zurück.

Das Bild, das ich hier gegeben habe, ist vielleicht etwas holzschnittartig. Im Großen und Ganzen aber ist es sicher so, dass die Linguisten allen an der Sprache Interessierten bislang wenig an lexikonfestem neuem Wissen vorlegen können - nicht weil es keine Fortschritte gegeben hat, sondern gerade, weil es viele Fortschritte gegeben hat. Warum erzähle ich all dies hier? Es erklärt zu einem wesentlichen Teil, weshalb das Programm einer Verbindung zwischen linguistischer und literaturwissenschaftlicher Analyse so wenig gediehen ist. Literarische Werke sind Hervorbringungen der menschlichen Sprachfähigkeit, und sie wirken, weil die umgekehrte, die rezeptive Seite der menschlichen Sprachfähigkeit sie verarbeiten kann. Es sind sprachliche Werke. Bei ihrer Produktion wie bei ihrer Rezeption spielen andere Bestandteile der 
menschlichen Kognition als das Sprachvermögen eine Rolle; dies ist aber bei anderen, nicht-ästhetischen Hervorbringungen der Sprache - einem Alltagsgespräch, einem Aufsatz, einer Sportreportage, nicht anders. Was immer ausgedrückt wird und was immer verstanden wird, hängt von zwei Arten der Information ab. Dies ist zum einen das sprachliche Wissen, also die Kenntnis der Wörter und der Regeln, nach denen sie sich zu größeren Einheiten, zu Sätzen und zu ganzen Texten, zusammenfügen lassen; zum anderen ist es das ganze kontextuelle Wissen von Sprecher und Hörer, Lesendem und Schreibendem - das, was sie dem zuvor Gesagten entnehmen können, was ihnen ihre Wahrnehmung über die jeweilige Situation sagt, was Teil ihres allgemeinen Weltwissens ist. Wie jemand „Ist zwivel herzen nachgebur, daz muoz der sele werden sur" versteht, hängt von der Kenntnis der Sprache und von einer Menge sonstigem - von Leser zu Leser höchst unterschiedlichem - Sachwissen ab; dies ist aber bei keiner Hervorbringung der Sprache, und sei sie noch so banal, wesentlich anders, und wie dies im Prinzip funktioniert, das müsste die Linguistik sagen oder jedenfalls sagen können.

Die Literaturwissenschaft befasst sich mit sprachlichen Werken einer bestimmten Art. Sie muss erklären können, was diese von anderen sprachlichen Werken unterscheidet, sie muss erklären können, was das einzelne sprachliche Kunstwerk von einem anderen sprachlichen Kunstwerk unterscheidet, sie muss das Schöne und das Erhabene und das Besondere im Schönen und im Erhabenen erklären. Die Linguistik kann dies nicht; es ist auch nicht ihre Aufgabe. Sie müsste aber eine Grundlage dazu bereitstellen können, eine Aufgabe, die sie bislang nicht gelöst hat.

\section{Vom Nutzen der Formalisierung}

\subsection{Wie ist es?}

Weiter oben habe ich eine Stelle aus einer syntaktischen Theorie zitiert, in der es um die Definition der Operation „Move" geht. Der Leser wird sie mit Interesse zur Kenntnis genommen haben. Der ungenannte Verfasser hat seinen Stolz in diese Definition gesetzt, aber andere erfüllt sie mit Unverständnis, Abneigung, ja Widerwillen. $\mathrm{Zu}$ diesen andern zählen nicht nur die Literaturwissenschaftler, sondern auch viele Sprachwissenschaftler. Die Fraktionierung, von der im vorigen Abschnitt die Rede war, hat sich in die Sprachwissenschaft selbst fortgesetzt. Dies hat wiederum viele Gründe; wenn sich eine Disziplin entwickelt, und das hat die Linguistik in den letzten dreißig Jahren getan, dann ist es nicht ungewöhnlich, dass sie sich zunehmend 
in Teilbereiche auflöst; wir haben dies oben am Beispiel der Philosophie erörtert. Hier will ich nur auf einen dieser Gründe eingehen, eben die Verwendung formaler Methoden und die damit einhergehende Esoterik.

Am Ausgang stehen zwei eigentümlich gegenläufige Tatsachen. Die erste dieser Tatsachen ist die folgende:

Formalisierungen signalisieren einen relativ hohen Rang in der Hackordnung des Faches.

Dieser Umstand äußert sich in drei recht unterschiedlichen Haltungen der Betroffenen:

1. Jene, die ihre Einsichten formal darstellen, glauben daran, dass dies die eigentlich wissenschaftliche Methode ist; andere Darstellungsformen sind zumindest unterlegen, vielleicht gar nur Vorstufen der wissenschaftlichen Aufarbeitung selbst.

2. Jene, die solche Methoden nicht verwenden, fühlen sich unterlegen; diese Haltung kann unterschiedliche Formen annehmen - von der leicht resignierten Einsicht ,ich kann's nicht recht" bis zu schwerer Traumatisierung.

3. Schließlich gibt es jene, die aus eben diesem Unterlegenheitsgefühl mit Abneigung reagieren und formale Methoden für * erklären (für * kann man einige Epitheta aus den niederen Stilschichten einsetzen).

All dies scheint mir recht offensichtlich, auch wenn es selten direkt angesprochen wird. Die andere, dazu entgegengesetzte Tatsache ist weitaus weniger auffällig:

Es gibt außer der modernen Linguistik keine empirische Wissenschaft, die ihre Erkenntnisse zu formalisieren sucht - es sei denn, es geht um irgendwelche numerischen Verhältnisse.

Man stelle sich vor, ein Biologe sähe sich, aus Gründen der Präzision und Explizitheit, veranlaßt, vom üblichen schlechten Englisch abzulassen und zu schreiben:

,? $\mathrm{x}(\mathrm{N}(\mathrm{x}) \mathrm{C}>\mathrm{P}(\mathrm{x})$, where $\mathrm{N}=$ neurone \& $\mathrm{P}=$ has at least one axone"

Seine Fachkollegen würden ihn für einen Wahnsinnigen halten. Ich denke auch nicht, dass einem Chemiker, einem Geologen oder einem Genetiker je der Gedanke gekommen ist, seine Erkenntnisse zu formalisieren. Dies besagt nicht, dass in diesen 
Disziplinen oder gar in der Physik, der Drosophila der Wissenschaftstheorie, nicht Formeln verwendet würden. Aber diese tauchen immer dann auf, wenn es um die präzisere Formulierung irgendwelcher quantitiver oder sonstwie numischer Verhältnisse geht: $\mathrm{e}=\mathrm{mc}^{2}$ oder $\mathrm{h}=6,625 \times 10^{\prime 34}$; dazu zähle ich hier auch geometrische Angaben, wie beispielsweise die Kepler'schen Gesetze (obwohl Kepler selbst sie nicht in Formeln ausgedrückt hat). Die vergleichsweise wenigen Linguisten, die quantitive Untersuchungen anstellen, können daher ebenso wenig der Formeln entraten wie die Physiker oder die Chemiker, wenn sie den Ionenfluss oder das Gasgleichgewicht beschreiben. Aber brauchen es die anderen, und wozu sind sie so total anders als die Forscher in den anderen empirischen Wissenschaften? Dies ist schwer zu beantworten. Vielleicht ist es hilfreich zuzuschauen, wie die Linguistik überhaupt zu den formalen Methoden gekommen ist.

\subsection{Wie ist es dazu gekommen?}

Mir scheint, man kann drei - teils zusammenhängende - Ursprünge erkennen, die zu den derzeitigen Formelwäldern geführt haben.

A. Die erste Linie geht auf die Entwicklung der modernen Logik zurück; sie lässt sich durch Namen wie Frege, Russell, Carnap, Tarski, Montague beschreiben, um nur einige der wichtigsten Exponenten zu nennen. Diese Linie hat zur Entwicklung der formalen Semantik geführt; die Syntax spielt dabei, wenn überhaupt, nur eine untergeordnete Rolle, andere Teilgebiete der Linguistik, etwa die Phonologie oder die Morphologie, noch weniger.

B. Die zweite Linie beginnt mit der Übertragung algebraischer Methoden, wie sie beim Studium formaler Sprachen verwandt werden, auf die Syntax natürlicher Sprachen. Diese Entwicklung setzt Mitte der Fünfzigerjahre mit Chomsky, Schützenberger, Miller und anderen ein und hat zumindest für ein Jahrzehnt die generative Grammatik maßgeblich geprägt; ich deute sie hier nur durch Begriffe wie „kontextfreie Grammatiken", ,weak generative power", „Lernbarkeit von Typ-1Sprachen" an. Diese Linie hat zur formalen Syntax geführt.

C. Die dritte Entwicklungslinie hat mit der maschinellen Verarbeitung natürlicher Sprache zu tun, kurz gesagt, mit der Computerlinguistik. Programme müssen in einer formalen Sprache geschrieben sein. Ebenso aber müssen die linguistischen Fakten formal ausspezifiziert werden, wenn man sie für die syntaktische Analyse, für die maschinelle Übersetzung, für Dialogsysteme und dergleichen verwenden will. Diese 
Linie hat daher primär auch zur formalen Syntax geführt; allerdings sind die tatsächlich verwandten formalen Grammatiken in aller Regel etwas anderer Natur als die unter 2. angesprochenen. Sie sind wesentlich stärker an der praktischen Brauchbarkeit als an prinzipiellen Fragen ausgerichtet: das System muss hinterher laufen, und ob die Beschreibung den unterschiedlichsten Adäquatheitsbedingungen des Linguisten genügt, ist zunächst einmal eine nachgeordnete Frage.

Es gibt viele Überschneidungen zwischen diesen drei Ursprüngen. Das will ich hier nicht weiter verfolgen, sondern mich der Frage zuwenden, was denn der Nutzen dieser drei Arten der Formalisierung für den Gegenstand der Linguistik - die Beschreibung und Erklärung der menschlichen Sprachfähigkeit und ihrer konkreten Manifestationen -ist.

\subsection{Was nutzen formale Methoden?}

Bei den unter Punkt C. genannten Entwicklungen ist die Antwort am einfachsten. Vielleicht kommt man einmal dahin, dass die Rechner auch natürliche Sprachen verstehen; aber selbst dann muss ihnen deren Syntax und Semantik formal aufbereitet werden. Dazu braucht man HPSG oder irgendein anderes formales System, und dasselbe gilt für die semantische Seite. Allerdings geht es dabei nicht um die wissenschaftlichen Ziele der Linguistik, sondern um Anwendungen auf dem Computer. Für die rein wissenschaftliche Untersuchung der menschlichen Sprachfähigkeit und ihrer Gesetzlichkeiten folgt daraus nichts, weder, dass man solche Methoden verwenden soll, noch, dass man sie nicht verwenden soll.

Formale Syntaxen im Sinne von B. sind ganz aus der Mode geraten; im Rahmen der generativen Grammatik selbst fuhren sie seit Ende der Sechzigerjahre ein Aschenputteldasein. Darüber darf die häufige Verwendung von Strukturbäumen und auch von Definitionen wie der in Abschnitt 3 zitierten nicht hinwegtäuschen. Weder im Modell der „Aspects" noch im „Principles-and-Parameters"-Rahmen noch im Minimalistischen Programm ist die Syntax formal ausbuchstabiert. Es handelt sich im Wesentlichen um sehr informelle Charakterisierungen mit gelegentlichen präzisen formalen Ausdifferenzierungen. Einer der Gründe für diese Entwicklung - sicher jedoch nicht der einzige - ist die Wendung von „Regeln" zu „Prinzipien", vielleicht auch ganz einfach die oft apostrophierte Verschiebung des Interesses von der präzisen und expliziten Beschreibung einzelsprachlicher Fakten zu übergreifenden „Erklärungen". Man mag dies bedauern oder nicht - wo die generative Grammatik unserer Tage for- 
mal scheint, handelt es sich, ganz anders als beispielsweise in der formalen Semantik, großenteils um Abkürzungen und Scheinformalismen. Wer es nicht glaubt, möge einfach einmal versuchen, eine grammatische Analyse in diesem Rahmen in eine Anwendung umzusetzen, bei der Explizitheit und Präzision unabdinglich sind, beispielsweise in eine maschinelle syntaktische Analyse oder auch in ein System, mit dem getestet wird, ob die von dem jeweiligen Grammatikfragment beschriebenen Strukturen wohlgeformt sind. Es ist nicht unmöglich, aber nur mit einem erheblichen Aufwand und unter Einbezug tatsächlicher formaler Systeme.

Diese Praxis scheint mir selbst das schlechteste aller Vorgehen, und das aus zwei Gründen. Sie ist zum einen schwierig, esoterisch und für den Außenstehenden, manchmal sogar für den Innestehenden, unverständlich, ohne doch den Vorzug formaler Systeme, nämlich ihre Präzision und Explizitheit, aufzuweisen. Zum anderen führt sie leicht dazu, die Probleme zu vernebeln. Ein Beispiel dafür ist die Neigung, allerlei grundlegende Gesetzlichkeiten in Begriffen der Baumgeometrie zu erklären. Die Relation „c-command" ist dafür das bekannteste Beispiel; ein anderes ist die Unterscheidung zwischen ,akkusativischen" Verben und „ergativen" Verben, bei denen das „Subjekt" an der Oberfläche auf einer anderen Strukturebene das „direkte Objekt" ist. Da dieser Punkt vielleicht nicht unmittelbar einleuchtend ist, will ich ihn kurz erläutern. Die Frage ist, sehr vereinfacht gesagt, „was die Striche im Baum bedeuten", oder etwas anders formuliert, weshalb man in bestimmten Fällen Dominanz ansetzt. Die Striche kommen ja nicht von selbst in den Baum. In einem rein formalen System reflektiert der Baum nur die Anwendung bestimmter formaler Regeln, die ihrerseits nach rein distributioneilen Kriterien konzipiert sind: es muss halt unten eine zulässige Kette stehen. In einem solchen System ist es egal, ob man schreibt: „VP C> V NP" oder „VP C> V X und X C> NP"; das Ergebnis ist dasselbe, die Bäume sind verschieden. In der Linguistik aber möchte man sinnvolle Bäume, d.h. ein Strich soll nicht nur die Ableitungsgeschichte repräsentieren, sondern deutbar sein, beispielsweise als „Rektion" bei ein Buch lesen oder bei einem Freund gehören, hingegen als adverbiale Modifikation bei gerne lesen. Deshalb muss man über die Baumgeometrie hinaus rechtfertigen, weshalb beispielsweise etwas als „direktes Objekt" oder als „Subjekt" angesehen werden soll. Zwar kann man beispielsweise sagen, ein direktes Objekt ist eine NP, die unmittelbar von V dominiert wird (oder so ähnlich); aber die Frage ist, wieso man diese NP dorthin gestellt hat.

Anders ist dies in der formalen Semantik, d.h. jenen formalen Systemen, die sich im Verfolg der formalen Logik entwickelt haben und deren systematische Anwendung auf Erscheinungen der natürlichen Sprache wir in erster Linie Richard Montague 
verdanken. Die Analyse semantischer Phänomene in diesem Rahmen ist präzise, wohldefiniert und im Prinzip auch klar zu verstehen - freilich nicht immer in der Praxis. Um es mit den leicht abgewandelten Worten eines mittelalterlichen Mönchs zu sagen (ich verdanke diesen Spruch, aber nicht die Abwandlung, Arnim von Stechow):

Wie der Fremde sich freut beim Anblick der Heimat,

der Seefahrer, gewahrt er des Hafens,

der Kämpfende, wenn der Sieg da ist,

der Händler, wenn der Gewinn sich einstellt,

der Kranke, wenn Gesundheit wiederkehrt,

So freut sich der Leser beim Anblick des Endes der Formel.

Steht dieser Aufwand in einem vernünftigen Verhältnis zum wissenschaftlichen Ertrag, d.h. zu einem besseren Verständnis der Bedeutung von einfachen und zusammengesetzten Ausdrücken? Da bin ich mir nicht sicher; vielleicht kommt es auf den Fall an. Jedenfalls gibt es einige Probleme, die zumindest mich zu einer ambivalenten Haltung veranlassen.

1. Während die formale Semantik im Prinzip eine sehr präzise Wissenschaft ist, ist sie in der Praxis oft eine erhebliche Schlamperei. Die reicht von kleinen Druckfehlern, die nur deshalb nicht banal sind, weil sie den nicht ganz so souveränen Leser in den Wahnsinn treiben, bis zu völligen Absurditäten bei manchen Autoren, die glauben, sich auf diesem Gebiet versuchen zu müssen, ohne es wirklich zu beherrschen. Sie legen etwas Bühnenschmuck an, um sich in der Hackordung hoch zu platzieren. Nun wäre es unbillig, die schlechte Praxis zum Maßstab für den wissenschaftlichen Nutzen einer bestimmten Methode oder einer bestimmten Beschreibungssprache zu nehmen. Wenn eine Methode schlecht angewandt wird, kann die Methode nichts dafür. Es ist jedoch ein eminentes Problem für die Praxis der Wissenschaft, nicht anders als die falsche oder gar absurde Anwendung statistischer Methoden in anderen Disziplinen. Nur lässt man da eine schlechte Anwendung nicht so einfach durchgehen.

2. In zahllosen Abhandlungen finden sich semantische Formeln, die zwar nicht falsch, aber schlichtweg Dekoration sind. Sie wiederholen noch einmal, was zuvor schon unmissverständlich in klarer Prosa gesagt wurde. Ich habe mich weiter oben mit dem biologischen Beispiel ,? x (N (x) C> P (x), where $\mathrm{N}=$ neurone \& $\mathrm{P}=$ has at least one axone" ein wenig darüber mokiert; jeder Biologe würde sich dabei an den Kopf fassen.

${ }^{1}$ Im Original heißt die letzte Zeile „,So freut sich der Autor beim Anblick des Endes des Buches". 
In linguistischen Arbeiten wimmelt es von solchen Beispielen, weil die Autoren aus irgendeinem Grund meinen, sie müssten das Ganze noch einmal in einem Ausdruck der elementaren Prädikatenlogik sagen, damit es wirklich wissenschaftlich ist. (Ich weiß, ich mache mich hier nicht beliebt, aber ich gebe auch keine Beispiele; vielleicht habe ich das alles ja nur geträumt).

3. Traditionell hat man unter „Semantik" die Lehre von der Bedeutung elementarer Ausdrücke verstanden: sie war „lexikalische Semantik". Die formale Semantik befasst sich mit ganz wenigen Ausnahmen nur mit der Frage, wie sich die Bedeutung komplexer Ausdrücke aus der seiner Bestandteile ergibt: sie ist kompositioneile Semantik. Die Ausnahmen gelten zum einen der Bedeutung bestimmter funktionaler Morpheme (etwa bei der Analyse des Tempus) und zum anderen gewissen strukturellen Eigenschaften in der Semantik komplexer Verben (etwa bei der semantischen Dekomposition im Sinne Dowtys). Nun sind dies bedeutende Leistungen, die ich auf keinen Fall herunterspielen möchte, ganz im Gegenteil. Aber man muss sie im Verhältnis zur gesamten Aufgabe sehen, vor die sich der Semantiker gestellt sieht. Dies ist die Aufgabe, die Bedeutung einfacher und zusammengesetzter Ausdrücke zu beschreiben. Wenn jemand die Bedeutung aller chinesischen Wörter kennen würde, aber keine einzige Regel, nach denen sich diese zu komplexen Bedeutungen zusammensetzen, dann würde man sagen, dass er wesentliche Teile des einschlägigen sprachlichen Wissens beherrscht. Wenn jemand die Bedeutung aller kompositioneilen Regeln des Chinesischen kennen würde, nicht aber die auch nur eines einzigen Wortes, dann würde man nicht sagen, dass er viel vom Chinesischen weiß. Ein Linguist, der das lexikalische Wissen beschreibt, hat einen großen Teil des sprachlichen Wissens abgedeckt; nicht so einer, der die kompositionale Semantik einer bestimmten Sprache beschrieben hat - so bewundernswert auch dies schon wäre.

4. Formale Sprachen und darunter eben auch jene, die in der formalen Semantik angewandt werden, haben einen bestimmten Ausdrucksreichtum, der sich aus der Art und Weise ergibt, wie diese Sprachen definiert sind. In der Aussagenlogik ist er sehr beschränkt, in der Prädikatenlogik schon wesentlich reicher und in den heute zur Verfügung stehenden Systemen recht beeindruckend. Aber vieles lässt sich in solchen Systemen nicht oder nur sehr schlecht ausdrücken, z.B. die Bedeutung des Satzes, den ich gerade schreibe, den der Leser gerade liest und von dem ich glaube, dass der Leser ihn ohne weiteres versteht. Dafür sind solche Systeme auch nicht gedacht. Aber man kann umgekehrt nicht bestreiten, dass dieser Satz, wie alle Sätze, eine klare, verständliche Bedeutung hat, die zu beschreiben letztlich das Ziel der linguistischen Analyse sein muss. Ich habe auch kein Rezept, wie dies gehen soll - aber wenn es 
gehen soll, dann nur, indem man den unendlichen Ausdrucksreichtum natürlicher Sprachen ausnutzt, ohne deshalb gleich in Konfusion und Vagheit zu verfallen.

Kommen wir nun noch einmal zum Ausgangspunkt dieser Überlegungen zurück. Dies war eine doppelte Tatsache, nämlich zum einen der Umstand, dass in der gegenwärtigen Linguistik Formalisierungen einen relativ hohen Rang in der Hackordnung signalisieren, und zum anderen der Umstand, dass dies eine ausgesprochene Besonderheit ist: Es gibt außer der modernen Linguistik keine empirische Wissenschaft, die ihre Erkenntnisse $\mathrm{zu}$ formalisieren sucht - es sei denn, es geht um irgendwelche numerischen Verhältnisse. Die Einstellung, die in Ersterem zum Ausdruck kommt, scheint mir absurd. Der Wert eines wissenschaftlichen Beitrags bemisst sich daran, ob er die Erkenntnis des Gegenstandes, um den es geht, voranbringt, nicht an der Anwendung einer bestimmten Methode oder einer bestimmten Art und Weise, diese Erkenntnis auszudrücken. Es ist unsinnig, von einem Evolutionsbiologen zu verlangen, er solle seine Ansichten gefälligst in einer formalen Sprache niederschreiben; umgekehrt kann ein solcher Biologe, etwa wenn es um populationsgenetische Gegebenheiten geht, vielleicht nur zum Ausdruck bringen, was er sagen möchte, wenn er sich dazu bestimmter mathematischer Ausdrücke bedient. Dann muss er sie verwenden. So, ganz genau so sollte es auch in der Linguistik sein.

\section{Envoi}

Es bliebe Vieles zu sagen, vor allem darüber, was man vielleicht anders machen könnte. Das will ich hier jedoch nicht tun, sondern nur noch auf einen nicht eben unwichtigen Punkt zu sprechen kommen. Bislang war eigentlich nur vom Überbau die Rede, nicht von der materiellen Grundlage der modernen Linguistik und ihrer Entwicklung in Deutschland seit Mitte der Sechzigerjahre. Und da ist es so, dass die Forschung in dieser Zeit im Wesentlichen von der Deutschen Forschungsgemeinschaft getragen wurde. Neue Universitätsstellen hat es in nennenswertem Maße nur zu Anfang der Siebzigerjahre gegeben, und da auch nicht für die Sprachwissenschaft im besonderen. Gleichzeitig sind die Studentenzahlen und damit die Belastung in der Lehre enorm gestiegen. Die neuere linguistische Forschung, die sich in mancher Hinsicht vielleicht doch von der übrigen Entwicklung in Deutschland abhebt, wurde im Wesentlichen über Drittmittel getragen, und dies wiederum heißt vor allem über Schwerpunkte, Forschergruppen, Sonderforschungsbereiche. Wir sollten wissen, wem wir unsere Stellen zu verdanken haben. 